\title{
Anafilaxia ao ácido poli-L-láctico
}

\author{
Poly-L-lactic acid anaphylaxis
}

Gil Bardini-Alves ${ }^{1}$, Natália Dal-Pizzol' ${ }^{1}$, Aline Vieira-Scarlatelli-Lima-Bardini1

\section{RESUMO}

Paciente feminina, 47 anos, previamente hígida, apresentou reação anafilática associada ao uso do ácido poli-L-láctico (PLLA). Imediatamente após a administração do bioestimulador, a paciente referiu edema de face que evoluiu para urticária generalizada, edema em membros inferiores e tremores. Posteriormente, apresentou edema de língua e dificuldade para falar. Teste de puntura com extratos de PLLA na concentração pura 1:1 e testes intradérmicos na diluição 1:10 e 1:100 mostrou-se positivo. Paciente negou cofatores no dia do procedimento e alergias prévias. O presente artigo descreve o primeiro caso da literatura de anafilaxia ao PLLA, onde se discute aspectos da reação anafilática e exames usados para o diagnóstico.

Descritores: Anafilaxia, urticária, hipersensibilidade a drogas, angioedema, fármacos dermatológicos.

\section{Introdução}

A anafilaxia é definida como uma reação de hipersensibilidade aguda, de rápida evolução, grave e potencialmente fatal. Na maioria das vezes é uma reação mediada por lgE e ocorre em pessoas previamente sensibilizadas quando são expostas novamente ao antígeno sensibilizador. Ela causa repercussões multissitêmicas, que incluem o envolvimento de pele e mucosas (cerca de $90 \%$ dos episódios), aparelho respiratório (40 a 60\%), trato gastrointestinal (25 a $30 \%$ ), sistema cardiovascular (30 a $35 \%$ ) e sistema nervoso central (10 a 15\% dos episódios) ${ }^{1}$.

\section{ABSTRACT}

A previously healthy 47-year-old woman had an anaphylactic reaction caused by poly-L-lactic acid (PLLA). Immediately after the administration of the biostimulating agent, the patient reported facial edema, which progressed to generalized urticaria, lower extremity edema, and tremors. Then she had tongue edema and difficulties to talk. A prick test with pure PLLA extracts (1:1) and intradermic tests (1:10 and 1:100 dilutions) were positive. The patient denied cofactors on procedure day as well as previous allergies. This is the first case report of anaphylaxis due to PLLA and includes a discussion of aspects of the anaphylactic reaction and tests that were used to provide the diagnosis.

Keywords: Anaphylaxis, urticaria, drug hypersensitivity, angioedema, dermatologic agents.

O diagnóstico da anafilaxia é, eminentemente, clínico, sendo altamente provável quando um dos três critérios descritos na Tabela 1 é preenchido? A suspeita clínica e a identificação da etiologia são fundamentais para o manejo adequado dos pacientes durante episódio agudo, variando conforme idade e, sobretudo, local onde a reação ocorre. Para confirmação da etiologia, podem ser necessários exames complementares como dosagem da IgE específica, teste por puntura ou provocação. Mesmo com a baixa incidência de reações sistêmicas aos testes cutâ-

1. Universidade do Sul de Santa Catarina, Curso de Medicina - Tubarão, SC, Brasil.

Submetido em: 20/09/2020, aceito em: 11/07/2021.

Arq Asma Alerg Imunol. 2021;5(3):291-4.

http://dx.doi.org/10.5935/2526-5393.20210045 
neos $^{3}$, deve-se sempre avaliar o risco e o benefício antes de realizá-los.

O tratamento de primeira escolha para reação anafilática é a administração precoce de adrenalina intramuscular $(1 \mathrm{mg} / \mathrm{mL}$ em adultos e $0,01 \mathrm{mg} / \mathrm{kg}$ em crianças). Medidas de segunda linha incluem fluidoterapia endovenosa, $\beta 2$-agonistas, corticoterapia, uso de anti-histamínicos e medidas gerais, como posicionar o paciente em decúbito dorsal com membros inferiores elevados (Trendelemburg) e a oxigenoterapia 4 .

A aplicação de preenchedores faciais, como o ácido hialurônico, o polimetilmetacrilato (Bellafill ${ }^{\circledR}$ ), substâncias à base de hidroxilapatita de cálcio (Radiesse ${ }^{\circledR}$ ) e o ácido poli-L-lático (Sculptra ${ }^{\circledR}$ ) é amplamente utilizada atualmente na abordagem terapêutica do rejuvenescimento. O ácido poli-Lláctico (PLLA) é um novo procedimento dermatológico usado para tratamento e prevenção da lipoatrofia e do envelhecimento facial. Trata-se de um polímero biocompatível, reabsorvível e imunologicamente inerte, que age estimulando a produção de fibroblastos e, consequentemente, de fibras de colágeno, aumentando a tensão da pele e levando à atenuação dos sulcos, rugas e marcas de expressão 5 . $\mathrm{O}$ ácido é totalmente eliminado do organismo em aproximadamente 18 meses $^{6}$. As reações adversas mais comuns relacionadas ao PLLA são no local de injeção do produto ${ }^{7}$. No entanto, há descrição de um relato de caso de amaurose ${ }^{8}$ e um de angioedema ${ }^{9}$ após aplicação do Sculptra ${ }^{\circledR}$.

O objetivo deste artigo foi relatar um caso de reação anafilática associada ao uso de ácido poli-Lláctico e correlacionar com dados da literatura.

\section{Relato de caso}

Paciente feminina, previamente hígida, 47 anos vem à consulta referindo, há cinco semanas, reação anafilática após primeiro procedimento com PLLA. Para o procedimento, foi utilizado lidocaína $2 \%$ e clorexidine $2 \%$ além do bioestimulador de colágeno. Imediatamente após a administração, a paciente referiu edema em face, que evoluiu para urticária generalizada, edema em membros inferiores e tremores. Paciente foi medicada com prednisolona oral e betametasona intramuscular, evoluindo com edema de língua e dificuldade para falar. Paciente, então, foi encaminhada ao pronto atendimento sendo administrada dexametasona endovenosa, adrenalina e prometazina intramuscular, evoluindo com melhora clínica e alta com prednisolona oral.

\section{Tabela 1}

Critérios para o diagnóstico de anafilaxia

1. Início agudo da doença (minutos ou horas) com envolvimento da pele, mucosas ou ambos (como urticária generalizada, prurido facial, edema de lábios...), e pelo menos um dos seguintes:

a) Pressão arterial reduzida ou sintomas associados de disfunção orgânica (hipotonia, síncope, incontinência...);

b) Comprometimento respiratório (dispneia, sibilos, estridor, hipoxemia...).

2. Dois ou mais dos seguintes sintomas ocorrendo rapidamente após exposição a um alérgeno provável:
a) Envolvimento de pele e mucosas;
b) Comprometimento respiratório;
c) Pressão arterial reduzida ou sintomas associados de disfunção orgânica;
d) Sintomas gastrintestinais persistentes.

3. Queda aguda da pressão arterial após exposição a um alérgeno conhecido:
a) Lactentes e crianças: PA sistólica baixa, conforme a idade do paciente, ou uma queda na PA sistólica basal maior que $30 \%$;
b) Adultos: PA sistólica menor que $90 \mathrm{mmHg}$ ou queda maior que $30 \%$ na PA sistólica basal. 
Paciente negava cofatores (exercício físico, uso de anti-inflamatórios e ingestão de bebidas alcoólicas) no dia do procedimento, bem como, alergias prévias e uso regular de medicações.

Os exames laboratoriais de dosagem de triptase sérica, hemograma, dosagem de IgE específica para clorexidine e látex estavam normais. Procedeu-se, então, à realização do teste cutâneo por puntura (prick test) com extratos de PLLA e lidocaína 2\% sem vasoconstrictor, ambos em sua forma pura (não diluídos). O resultado do prick-test para PLLA evidenciou-se positivo (pápula $4 \times 4 \mathrm{~mm}$ e eritema $15 \times 15 \mathrm{~mm}$ ) e negativo para lidocaína (Figura 1). O teste de provocação cutânea com luva de látex (use-test) foi negativo.

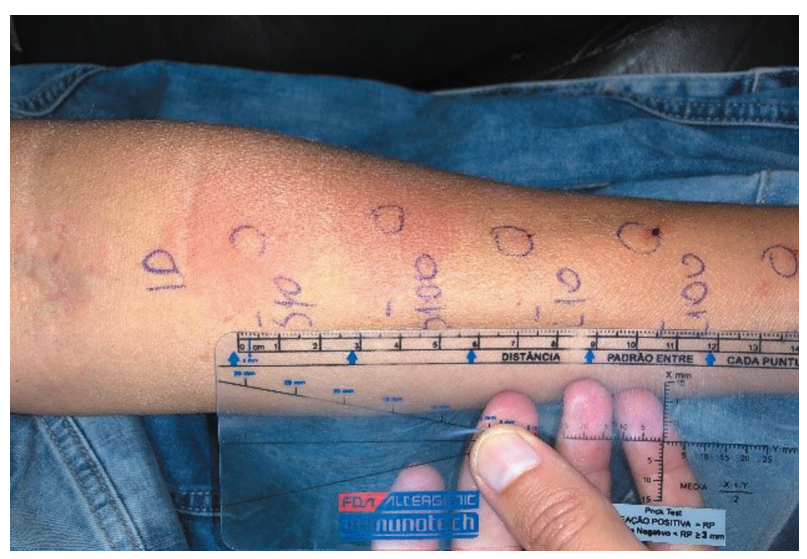

\section{Figura 1}

Resultado positivo do prick test, com pápula de $4 \times 4 \mathrm{~mm}$ e eritema de $15 \times 15 \mathrm{~mm}$, e negativo para lidocaína. Mesmo com a baixa incidência de reações sistêmicas aos testes cutâneos, deve-se sempre avaliar o risco versus o benefício antes de realizá-los

Por não haver um teste cutâneo padronizado para PLLA, foi optado por realizar teste intradérmico nas diluições 1:10 e 1:100 para confirmar os achados encontrados no prick test. O teste foi positivo (incremento de $3 \mathrm{~mm}$ além da pápula inicial associado a um eritema maior que $20 \mathrm{~mm}$ de diâmetro) em ambas diluições (Figura 2). O teste intradérmico para lidocaína nas diluições 1:10 e 1:100 e o controle negativo com solução salina $0,9 \%$ foram negativos.

O mesmo teste cutâneo com PLLA foi negativo quando realizado em outros dois pacientes controles que já haviam utilizado anteriormente a substância sem relato de reações alérgicas.

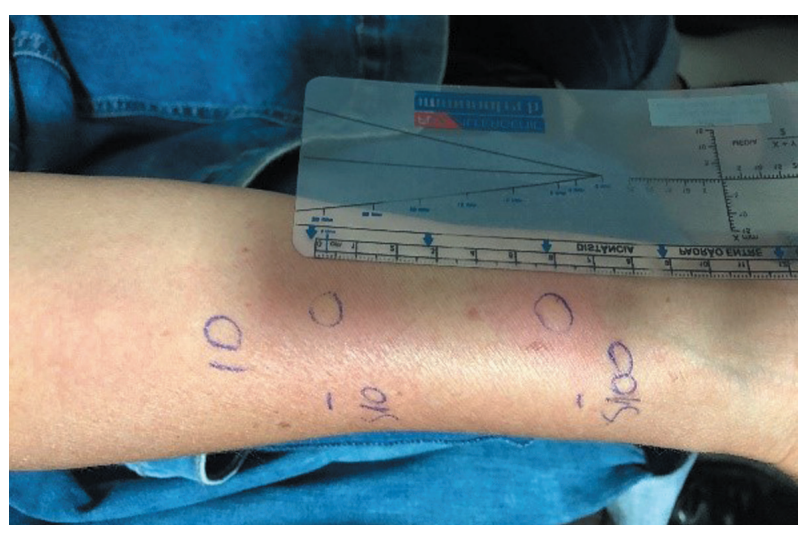

Figura 2

Teste intradérmico positivo nas diluições 1:10 e 1:100 de PLLA, com incremento de $3 \mathrm{~mm}$ além da pápula inicial associado a um eritema maior que $20 \mathrm{~mm}$ de diâmetro em ambas diluições

Diante da história clínica compatível e resultados dos exames, a conclusão é que se trata de uma reação anafilática secundária ao PLLA.

\section{Discussão}

Os preenchimentos injetáveis estão aumentando em popularidade como uma opção pouco invasiva para pacientes que buscam o rejuvenescimento facial. Nos Estados Unidos, a procura por procedimentos cosméticos minimamente invasivos aumentou $48 \%$ de 2009 para $2019^{10}$. O risco de reações adversas ao seu uso varia conforme a substância utilizada e o local de aplicação. Nos preenchimentos à base de ácido hialurônico, que atualmente são os mais utilizados, infecção e inchaço são as complicações mais comumente relatadas ${ }^{11}$. Complicações graves como cegueira e necrose da pele estão mais relacionadas à aplicação de hidroxilapatita de cálcio (Radiesse $\left.{ }^{\circledR}\right)^{11}$; além disso, um caso de anafilaxia com prurido difuso, urticária, edema facial e edema de glote após a aplicação dessa substância também já foi relatado ${ }^{12}$.

O PLLA possui reações adversas mais frequentemente na região de injeção do produto e incluem reiteradamente pápulas e nódulos apenas palpáveis, mas não visíveis ${ }^{7,11}$. Os nódulos também são a complicação mais comum em longo prazo, tendo geralmente resolução espontânea em 24 meses $^{13}$. Outras reações locais menos comuns são equimoses, hematomas, edema e granulomas ${ }^{7}$. Como complicações graves, um caso de amaurose ${ }^{8}$ e um caso de angioedema ${ }^{9}$ após PLLA já foram descritos. No presente trabalho, apresentamos um provável quadro 
de reação anafilática ao ácido poli-L-láctico que, até o momento, não há relatos na literatura.

$\mathrm{Na}$ paciente descrita a anafilaxia apresentada foi IgE mediada, já que os resultados dos testes cutâneos de hipersensibilidade imediata para a substância foram positivos. Foi optado por realizar o teste cutâneo diagnóstico, visto que incidência de reações sistêmicas aos testes cutâneos é baixa ${ }^{3}$ e o risco de a paciente ter outra reação anafilática pelo contato com a substância sensibilizadora durante a vida é muito maior. Com isso, visto não haver um teste cutâneo padronizado para PLLA, foi realizado o teste intradérmico com duas diluições diferentes associado ao teste controle em duas pessoas saudáveis. A paciente negava procedimentos prévios utilizando PLLA, portanto, podemos inferir que outra substância provocou a sensibilização. O Sculptra ${ }^{\circledR}$ é apresentado na forma de pó liofilizado e contém micropartículas de PLLA, que é produzido a partir da fermentação do ácido láctico no milho, manitol não pirogênico e croscarmelose sódica7. Ambas substâncias são amplamente usadas pela indústria farmacêutica e podem ter causado a sensibilização primária.

Como se trata do primeiro caso descrito de anafilaxia ao PLLA, futuros trabalhos são necessários para avaliar qual foi o mecanismo de sensibilização, tendo como hipóteses: outro componente presente no bioestimulador ou sensibilizações cruzadas com outros alérgenos.

\section{Referências}

1. Lockey RF, Kemp SF, Lieberman PL, Sheikh A. Anaphylaxis. in: Pawankar R, Canonica GW, Holgate S, Lockey R, Blaiss M (eds). World Allergy Organization (WAO) White Book on Allergy. Update 2013. WAO; 2013. p.48-53.

2. Sampson HA, Muñoz-Furlong A, Campbell RL, Adkinson NF Jr, Bock SA, Branum A, et al. Second symposium on the definition and management of anaphylaxis: summary report. J Allergy Clin Immunol. 2006;117:391-7.
3. Bernd LA, Solé D, Pastorino AC, Prado EA, Castro FFM, Rizzo $\mathrm{MC}$, et al. Anafilaxia: guia prático para manejo. Rev bras alerg imunopatol. 2006;29(6):283-91.

4. Sarinho ECS, Chong Neto HJ, Antunes AA, Pastorino AC, Porto Neto AC, Kuschnir FC, et al. Guia Prático de Atualização em Anafilaxia - Sociedade Brasileira de Pediatria. Rio de Janeiro: SBP;2016.

5. Lowe NJ. Optimizing poly-L-lactic acid use. J Cosmet Laser Ther. 2008;10(1):43-6.

6. Rendon MI. Long-term aesthetic outcomes with injectable poly-L-lactic acid: observations and practical recommendations based on clinical experience over 5 years. J Cosmet Dermatol. 2012;11(2):93-100.

7. Santos CAM Filho, Santos TC, Juberto Rodrigues AP, Gonzaga da Cunha M. Ácido poli-láctico: um agente bioestimulador. Surg Cosmet Dermatol. 2013;5(4):345-50.

8. Roberts SA, Arthurs BP. Severe visual loss and orbital infarction following periorbital aesthetic poly-L-lactic acid (PLLA) injection. Ophthal Plast Reconstr Surg. 2012;28(3):e68-70.

9. Guardiani E, Davison SP. Angioedema after treatment with injectable poly-L-lactic acid (Sculptra). Plast Reconstr Surg. 2012;129(1):187e-189e.

10. American Society of Plastic Surgeons:2009 Plastic Surgery Statistics Report [Internet]. Disponível em: https://www.plasticsurgery.org/ documents/News/Statistics/2009/plastic-surgery-statistics-fullreport-2009.pdf.

11. Rayess HM, Svider PF, Hanba C, Patel VS, DeJoseph LM, Carron $\mathrm{M}$, et al. A Cross-sectional Analysis of Adverse Events and Litigation for Injectable Fillers. JAMA Facial Plast Surg. 2018;20(3):207-14. doi: 10.1001/jamafacial.2017.1888.

12. Cohen JC, Reisacher W, Malone M, Sulica L. Severe systemic reaction from calcium hydroxylapatite vocal fold filler. Laryngoscope. 2013;123(9):2237-9. doi: 10.1002/lary.23762.

13. Ledon JA, Savas JA, Yang S, Franca K, Camacho I, NouriK. Nódulos inflamatórios após o uso de preenchimento de tecido mole: uma revisão dos agentes causadores, patologia e opções de tratamento. Am J Clin Dermatol. 2013;14(5):401-11.

Não foram declarados conflitos de interesse associados à publicação deste artigo.

Correspondência:

Gil Bardini-Alves

E-mail: gilbardini@yahoo.com.br 